\title{
Dipterofauna do Arquipélago de Fernando de Noronha (Pernambuco, Brasil)
}

\author{
Márcia Souto Couri ${ }^{1,2}$, Gabriel Pinto da Silva Barros ${ }^{1,3} \&$ Marcelo Pinheiro Orsini1 ${ }^{1,4}$
}

\begin{abstract}
${ }^{1}$ Museu Nacional, Universidade Federal do Rio de Janeiro, Quinta da Boa Vista, 20940-040, Rio de Janeiro-RJ, Brasil. mcouri@terra.com.br ${ }^{2}$ Bolsista de Produtividade em Pesquisa do Conselho Nacional de Desenvolvimento Científico e Tecnológico, CNPq.

${ }^{3}$ Bolsista PIBIC/UFRJ.
\end{abstract}

${ }^{4}$ Bolsista PIBIC/CNPq.

\begin{abstract}
Dipterofauna of Fernando de Noronha (Pernambuco, Brazil). This paper records some Diptera from the island of Fernando de Noronha (Pernambuco, Brazil), presenting the richness of species and their abundance. The material studied was collected in July 1973. Eight families of Diptera in a total of 11,515 specimens were studied. Among the families the following seven are new records: Calliphoridae, Muscidae, Fanniidae, Stratiomyidae, Sepsidae, Otitidae and Tabanidae. The first three and the Sarcophagidae (previously recorded) were identified to species level. The most abundant families were Sepsidae and Calliphoridae with more than $80 \%$ of the total collected, having Cochliomyia macellaria (Fabricius, 1775), as the dominant species.
\end{abstract}

KEYWORDS. Diptera; diversity; taxonomy.

RESUMO. Dipterofauna de Fernando de Noronha (Pernambuco, Brasil). Este trabalho registra alguns dípteros da ilha de Fernando de Noronha (Pernambuco, Brasil), apresentando a riqueza das espécies e sua abundância. O material examinado foi coletado em julho de 1973. Oito famílias de Diptera, em um total de 11.515 indivíduos foram estudadas. Entre as famílias encontradas as sete seguintes são novos registros: Calliphoridae, Muscidae, Fanniidae, Stratiomyidae, Sepsidae, Otitidae e Tabanidae. As três primeiras e os Sarcophagidae (previamente registrada) foram identificadas até o nível de espécie. As famílias mais abundantes foram Sepsidae e Calliphoridae com mais de $80 \%$ do total coletado, tendo Cochliomyia macellaria (Fabricius, 1775) como espécie dominante.

PALAVRAS-CHAVE. Diptera; diversidade; taxonomia.

$\mathrm{O}$ arquipélago de Fernando de Noronha está situado na costa brasileira a $360 \mathrm{~km}$ de Natal, $710 \mathrm{~km}$ de Fortaleza e $545 \mathrm{~km}$ de Recife, sendo administrado pelo Estado de Pernambuco. São ao todo 21 ilhas ou ilhotas de natureza vulcânica, tendo se originado a partir de uma erupção ocorrida há 12 milhões de anos, com a principal ilha detendo o nome do arquipélago, pois é a mais extensa com $17 \mathrm{~km}^{2}$. Clima Tropical, quente oceânico, de estações bem definidas. Pluviometria média de $1.300 \mathrm{~mm}$ anual, com maiores índices entre março e maio e estiagem entre agosto e janeiro e temperatura média de $25,4^{\circ}$ C. Ventos constantes, velocidade média $6,6 \mathrm{~m} / \mathrm{seg}$. com maiores intensidades entre julho e agosto. Umidade relativa bastante constante, com média anual de $81 \%$. A vegetação predominante em Fernando de Noronha é composta por espécies típicas do agreste nordestino, perdendo sua folhagem na estação seca. Em seu aspecto geral, a vegetação apresenta árvores nas áreas mais elevadas e arbustos nas superfícies mais planas (www.noronha.pe.gov.br, consultado em 09/10/2008).

Vários projetos envolvendo grupos animais estão sendo desenvolvidos no arquipélago, mas quase não há registros sobre a dipterofauna da região, salvo Ridley (1890) e Alvarenga (1962) que registraram cinco espécies das famílias Dolichopodidae, Culicidae, Sarcophagidae e Syrphidae (Tab. I). O material estudado por estes autores foi proveniente de expedições científicas realizadas na ilha de Fernando de Noronha respectivamente em 1887 e 1954.
Esse artigo tem a finalidade de registrar os dípteros existentes no arquipélago com base em amostras coletadas e sua abundância, dando ênfase as famílias Muscidae, Calliphoridae, Sarcophagidae e Fanniidae.

\section{MATERIALE MÉTODOS}

O material estudado é proveniente da coleta realizada pelo Sr. Olmiro Roppa em julho de 1973 na ilha de Fernando de Noronha. Os espécimes foram montados em alfinetes entomológicos e triados por família. Para a identificação das espécies de Calliphoridae, Fanniidae e Muscidae foram utilizadas respectivamente as chaves de Mello (2003), Albuquerque et al. (1981), Carvalho \& Couri (2002) e Couri \& Carvalho (2002). Os exemplares da família Sarcophagidae foram triados e identificados pela $\mathrm{Dr}^{\mathrm{a}}$ Cátia Antunes de Mello-Patiu (Museu Nacional, Rio de Janeiro). Não há dados adicionais de coleta junto ao material, porém sabe-se por bibliografia e comunicação pessoal de pesquisadores do Museu Nacional que Olmiro Roppa tinha o hábito de coletar com a armadilha do tipo Shannon. Devido à natureza do material coletado, supõe-se que matéria orgânica em decomposição e fezes tenham sido utilizadas como iscas.

Todo material estudado foi incorporado à coleção do Museu Nacional (MNRJ). 


\section{RESULTADOS EDISCUSSÃO}

Foram triados um total de 11,515 indivíduos e identificadas oito famílias: Calliphoridae, Fanniidae, Muscidae, Otitidae, Sarcophagidae, Sepsidae, Stratiomyidae e Tabanidae (Tab.I). Em termos de abundância de indivíduos, Sepsidae e Calliphoridae representaram a grande maioria do material, com $42,9 \%$ e $41,2 \%$ respectivamente, totalizando mais de $80 \%$ do material examinado. A seguir, a família Muscidae, a mais diversificada com seis espécies, representou $10 \%$ do total, enquanto Sarcophagidae com três espécies representou 5,2\% (Fig.1).

A família Fanniidae está representada por machos de Fannia pusio (Wiedemann, 1830) e fêmeas do grupo pusio. Os adultos do gênero Fannia são encontrados freqüentemente associados ao ambiente modificado pelo homem, com suas larvas criando-se em matéria orgânica em decomposição (Pont 1977).

A família Calliphoridae foi representada por Cochliomyia macellaria (Fabricius, 1775) e Lucilia eximia (Wiedemann, 1819). C. macellaria umas das principais espécies causadora de miíase secundária no Brasil foi a mais abundante ultrapassando os $99 \%$ da população de califorídeos. Três espécies do gênero Chrysomya Robineau-Desvoidy, 1830: $C$. megacephala (Fabricius, 1794), C. albiceps (Wiedemann, 1819) e C. putoria (Wiedemann, 1818) foram introduzidas e se dispersaram no Brasil na década de 70 (Guimarães et al. 1978; Prado \& Guimarães, 1982). Estas espécies competem com $C$. macellaria e L. eximia; assim, novas coletas na ilha, seriam interessantes para verificar a introdução das espécies de Chrysomya e uma conseqüente diminuição nas populações de C. macellaria e L. eximia.

A família Muscidae é a que representa maior diversidade e

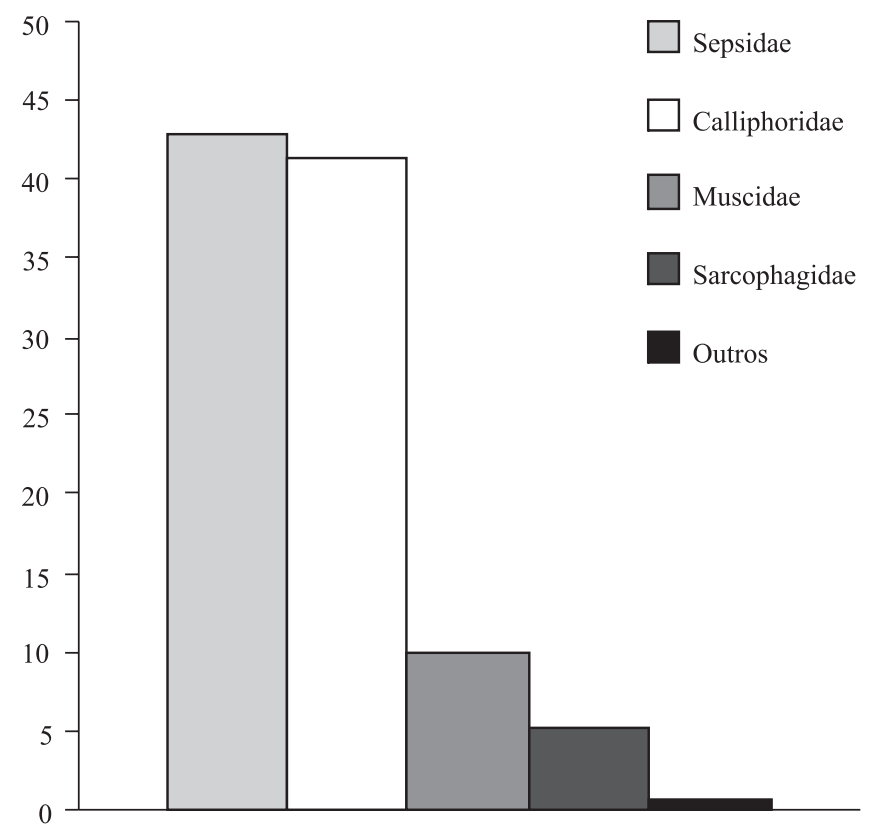

Fig. 1. Percentual de riqueza das famílias de Diptera no arquipélago de Fernando de Noronha (Pernambuco, Brasil). está representada por seis espécies (Fig. 2): Musca domestica Linnaeus, 1758; Atherigona orientalis Schiner, 1868; Brontaea quadristigma (Thomson, 1869); Brontaea debilis (Williston, 1896); Cyrtoneuropsis rescita (Walker, 1861) e Synthesiomyia nudiseta (Wulp, 1883). As espécies de Brontaea criam-se exclusivamente em fezes de mamíferos. Do total de Muscidae coletados, A. (Acritochaeta) orientalis representou quase $60 \%$. Esta espécie cria-se em matéria orgânica ou vegetal, sendo saprófaga ou carnívora facultativa. Há registros na literatura desta espécie atacando tecidos vegetais vivos, porém, a importância econômica deste gênero está mais relacionada com as espécies de Atherigona s.s. (sub-gênero Atherigona) tendo elas considerável importância como pragas de plantações de cereais.

Os Sarcophagidae estão representados por três espécies: Peckia chrysostoma (Wiedemann, 1830), Oxysarcodexia thornax (Walker, 1849) e Tricharaea occidua (Fabricius, 1794). Alvarenga (1962) registrou Nephochaetopteryx calida (Wiedemann, 1856) (=Sarcophaga calida) como única espécie da família presente no arquipélago, porém esta não foi encontrada nesta coleta.

Todas as espécies assinaladas são novos registros para o arquipélago de Fernando de Noronha, bem como as famílias Calliphoridae, Muscidae, Fanniidae, Stratiomyidae, Sepsidae, Otitidae e Tabanidae. Não foram assinaladas espécies endêmicas, sendo a maioria das espécies coletadas sinantrópicas e, portanto, associadas ao homem e ao ambiente modificado por ele. A origem das ilhas do arquipélago, associada a erupções vulcânicas, lhes confere ambientes singulares, diferentes do continente e propícios ao endemismo de espécies, como já verificado nas ilhas para outros grupos animais, entretanto, ainda não foram reconhecidos dípteros endêmicos neste arquipélago. Coletas futuras na ilha e a utilização de iscas da fauna local, como por exemplo, carcaças de animais marinhos, certamente complementarão esta lista que servirá para estudos comparativos.

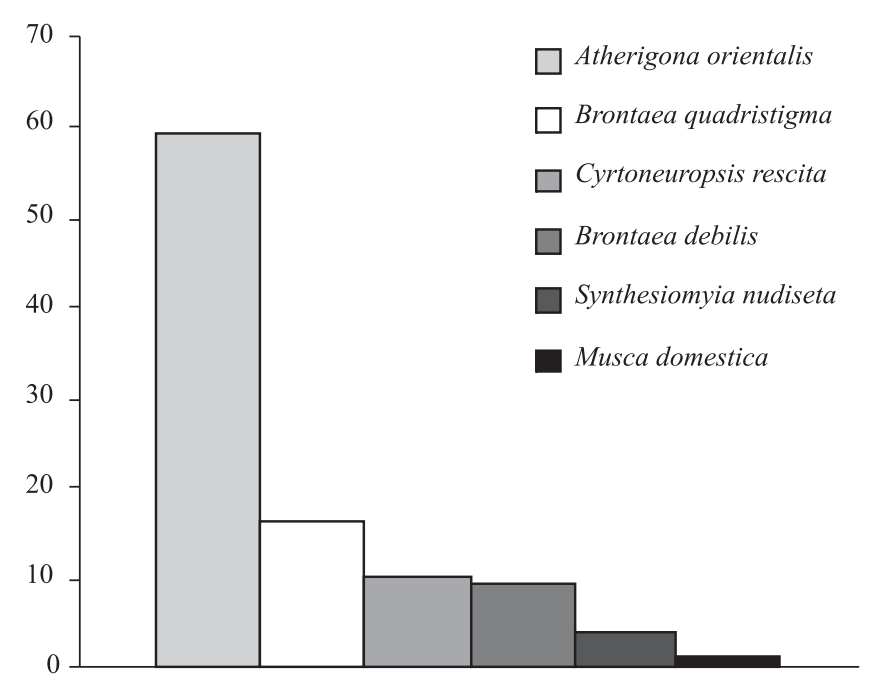

Fig. 2. Percentual de riqueza das espécies de Muscidae no arquipélago de Fernando de Noronha (Pernambuco, Brasil). 
Tabela I. Dipterofauna de Fernando de Noronha (Pernambuco, Brasil)

\begin{tabular}{|c|c|c|}
\hline Famílias/espécies & $\mathrm{N}^{\mathrm{o}}$ de indivíduos & Fonte \\
\hline \multicolumn{3}{|l|}{ Culicidae } \\
\hline Culex pipiens fatigans Wiedemann, 1828 & - & Alvarenga, 1962 \\
\hline Aedes taeniorhynchus (Wiedemann, 1821) & - & Alvarenga, 1962 \\
\hline \multicolumn{3}{|l|}{ Dolichopodidae } \\
\hline Condylostylos longicornis Fabricius, 1775 (=Psilopus metallifer Walter, 1849) & - & Ridley, 1890 \\
\hline \multicolumn{3}{|l|}{ Syrphidae } \\
\hline Temnocera vesiculosa (Fabricius, 1805) & - & Ridley, 1890 \\
\hline \multicolumn{3}{|l|}{ Sarcophagidae } \\
\hline Nephochaetopteryx calida (Wiedemann, 1856) (=Sarcophaga calida Wiedemann, 1846) & - & Ridley, 1890 \\
\hline Peckia crysostoma (Wiedemann, 1830$)$ & 24 & presente publicação \\
\hline Oxysarcodexia thornax (Walker, 1849) & 557 & presente publicação \\
\hline Tricharaea occidua (Fabricius, 1794) & 18 & presente publicação \\
\hline \multicolumn{3}{|l|}{ Sepsidae } \\
\hline Palaeosepsis sp. (Duda, 1926) & 4940 & presente publicação \\
\hline \multicolumn{3}{|l|}{ Calliphoridae } \\
\hline Lucilia eximia (Wiedemann, 1819) & 06 & presente publicação \\
\hline Cochliomyia macellaria (Fabricius, 1775) & 4743 & presente publicação \\
\hline \multicolumn{3}{|l|}{ Fanniidae } \\
\hline Grupo pusio (fêmeas) & 20 & presente publicação \\
\hline Fannia pusio (Wiedemann, 1830) & 03 & presente publicação \\
\hline \multicolumn{3}{|l|}{ Muscidae } \\
\hline Atherigona orientalis Schiner, 1868 & 687 & presente publicação \\
\hline Brontaea quadristigma (Thomson, 1869) & 189 & presente publicação \\
\hline Brontaea debilis (Williston, 1896) & 105 & presente publicação \\
\hline Cyrtoneuropsis rescita (Walker, 1861) & 117 & presente publicação \\
\hline Musca domestica Linnaeus, 1758 & 14 & presente publicação \\
\hline Synthesiomyia nudiseta (Wulp, 1883) & 45 & presente publicação \\
\hline Tabanidae & 01 & presente publicação \\
\hline Stratiomyidae & 02 & presente publicação \\
\hline Otitidae & 18 & presente publicação \\
\hline Outros & 26 & presente publicação \\
\hline Total & 11.515 & \\
\hline
\end{tabular}

Agradecimentos. A Dr ${ }^{a}$ Cátia Antunes de Mello-Patiu e Wallace Soares Faria (Museu Nacional, UFRJ) pela identificação dos espécimes da família Sarcophagidae e ao CNPq pelo apoio financeiro.

\section{REFERÊNCIAS}

Albuquerque, D. de O.; D. M. Pamplona \& C. J. B de Carvalho. 1981. Contribuição ao conhecimento dos Fannia R. D., 1830 da região neotropical (Diptera, Fanniidae). Arquivos do Museu Nacional 56: $9-34$.

Alvarenga, M. 1962. A entomofauna do arquipélago de Fernando de Noronha, Brasil. Arquivos do Museu Nacional 52: 21-26.

Carvalho, C. J. B de \& M. S. Couri. 2002. Part I. Basal groups: 17-132, In C.J.B de Carvalho ed., Muscidae (Diptera) of the Neotropical Region, Taxonomy. Curitiba, Editora Universidade Federal do Paraná: 287 p.
Couri, M. S. \& C. J. B de Carvalho. 2002. Part II. Apical groups: 133 257, In: C. J. B de Carvalho ed., Muscidae (Diptera) of the Neotropical Region, Taxonomy. Curitiba, Editora Universidade Federal do Paraná: 287 p.

Guimarães, J. H.; A. P. Prado \& A. X. Linhares. 1978. Three newly introduced blowfly species in southern Brazil (Diptera, Calliphoridae). Revista Brasileira de Entomologia 22: 53-60.

Mello, R. P. 2003. Chave para identificação das formas adultas das espécies da família Calliphoridae (Diptera, Brachycera, Cyclorrhapha) encontradas no Brasil. Entomologia y Vectores 10: $255-268$.

Pont, A. C. 1977. A revision of Australian Fanniidae (Diptera: Calyptrata). Australian Journal of Zoology. Supl. 51: 1-60.

Prado, A. P. \& J. H. Guimarães. 1982. Estudo atual de dispersão e distribuição do gênero Chrysomya Robineau-Desvoidy na região neotropical (Diptera, Calliphoridae). Revista Brasileira de Entomologia 26: 225-231.

Ridley, H. N. 1890. Notes on the Zoology of Fernando de Noronha. Journal of the Linnean Society of London 20: 473-56. 